\title{
Variation in the recorded incidence of amputation of the lower limb in England
}

\author{
N. Holman • R. J. Young • W. J. Jeffcoate
}

Received: 12 October 2011 /Accepted: 22 December 2011 /Published online: 8 March 2012

(C) Springer-Verlag 2012

\begin{abstract}
Aims/hypothesis The study aimed to explore the variation in recorded incidence of lower limb amputation in England. Methods The incidences of amputations in adults with and without diabetes were determined from hospital episode statistics over 3 years to 31 March 2010 and compared between the 151 Primary Care Trusts (PCTs) in England. Results There were 34,109 amputations, including 16,693 $(48.9 \%)$ in people with diabetes. The incidence was 2.51 per 1,000 person-years in people with diabetes and 0.11 per 1,000 person-years in people without (relative diabetes risk 23.3). Incidence varied eightfold across PCTs in people both with diabetes (range $0.64-5.25$ per 1,000 person-years) and without ( $0.03-0.24$ per 1,000 person-years). Amputations in people with diabetes varied tenfold - both major (range 0.22 2.20 per 1,000 person-years) and minor (range $0.30-3.25$ per 1,000 person-years). The incidences of minor and major amputations were positively correlated both in those with $(r=0.537, p<0.0005)$ and without $(r=0.611, p<0.0005)$ diabetes. Incidences of amputations were also correlated
\end{abstract}

\section{N. Holman}

Diabetes Health Intelligence,

Yorkshire and Humber Public Health Observatory,

York, UK

\section{R. J. Young}

Department of Diabetes and Endocrinology,

Salford Royal Hospital Foundation Trust,

Salford, UK

\section{W. J. Jeffcoate $(\square)$}

Foot Ulcer Trials Unit,

Department of Diabetes and Endocrinology,

Nottingham University Hospitals Trust,

City Hospital Campus,

Nottingham NG5 1PB, UK

e-mail: william.jeffcoate@nuh.nhs.uk between people with and without diabetes (total amputations $r=0.433, p<0.0005$; major amputations $r=0.528, p<0.0005$ ). There was a negative correlation between the incidence of amputation and estimated prevalence of ethnic Asians. No association was found between the PCT incidence of either total amputations and general population prevalence of social deprivation $(r=-0.138, p=0.092)$ or smoking $(r=0.137, p=0.096)$.

Conclusions/interpretation Variation in amputation incidence occurs across England. Because of the similarity in amputation variation between people with and without diabetes the variation may reflect generic differences in local healthcare delivery, although racial factors may also contribute.

Keywords Amputation · Complications · Diabetes · Diabetic foot · Gangrene · Neuropathy Peripheral arterial disease
Abbreviations
HES Hospital Episode Statistics
NDA National Diabetes Audit
NHS National Health Service
PCT Primary Care Trust
QOF Quality Outcome Framework

\section{Introduction}

Amputation of the lower limb is one of the most feared adverse health outcomes among people with diabetes. The result is frequently devastating in terms of social functioning and mood [1]. Amputation also poses a considerable cost to providers of healthcare, while the financial burden on the patient and their family can be enormous in countries that 
lack a comprehensive health service [2]. Reduction in the incidence of diabetes-related amputation is a major priority worldwide. Moves to reduce the incidence are likely to be most effective in those communities in which the baseline incidence is particularly high, and will be achieved mainly by improving access to effective primary care, with improved control of blood sugar and interventions to minimise the onset of complications such as peripheral arterial disease and neuropathy. Those with new diabetes-related ulceration of the foot should be referred promptly for expert assessment as an association has been demonstrated between time to expert referral and clinical outcome [3, 4].

Variation in access to effective healthcare may be one factor underlying the 8.6-fold variation in incidence of major amputation (an amputation performed above the ankle) observed between 306 healthcare regions in the USA [5]. Such variation in access should not apply to any great extent, however, in countries such as England that have a nationalised health service free to all at the point of delivery. Despite this, a fourfold variation in the incidence of total amputations (major plus minor) was observed between four localities in mid- to northern England in the 1990s [6]. More recently, the UK Atlas of Variation in Health Care has reported a twofold variation in the incidence of major amputation in type 2 diabetes between ten Strategic Health Authorities in England [7]. Such variation might reflect important differences in the structure of lower limb healthcare delivery. In order to explore the extent of this variation in more detail, we have examined the records of hospitals in England and have compared the incidence of amputations between the 151 Primary Care Trusts (PCTs) in England. PCTs are local health economies covering populations of 100,000 to 500,000 individuals, comprising all the patients registered with a geographically coherent group of general practitioners.

\section{Methods}

A search of Hospital Episode Statistics (HES) was made to identify all recorded lower limb amputations undertaken in adults (age 17 years and older) during admissions to all National Health Service (NHS) hospitals in England between 1 April 2007 and 31 March 2010. Traumatic amputations and those due to malignancy were included. People with diabetes were defined as those with a primary or secondary coded discharge diagnosis of diabetes (ICD-10 [www.who.int/classifications/icd/en/]: E10-14). A minor amputation was defined as one undertaken below the ankle (Office of Population Censuses and Surveys [OPCS] codes: X10-X11) and a major amputation was one above the ankle (OPCS codes: X091-X095, X098, X099). If there was more than one operation during a single admission, only one (the highest) was counted, but when amputations occurred during separate admissions they were counted as separate episodes. Patients were linked to PCTs by their home postcode. The prevalence of diabetes in the population of each PCT was derived from annual returns made by general practices to the Quality Outcome Framework (QOF), and was used to determine the incidence of amputations per 1,000 adults known to have the disease. Smoking prevalence estimates for the general population of each PCT were based on data from the Health Survey for England [8]. The average level of social deprivation for each PCT was measured using the indices of multiple deprivation 2007 [9]. Population estimates by ethnic group produced by the Office for National Statistics were used to calculate the proportion of the population from Asian ethnic groups and black ethnic groups for each PCT [10].

Associations between the rates of minor and major amputations in people with diabetes and people without diabetes, as well as between the rates of amputation, smoking prevalence, deprivation and the proportion of the population from Asian and black ethnic groups were analysed using Spearman's rank correlation coefficient. To assess categorical variables, $\chi^{2}$ tests were used. The ratio of major to minor amputations was calculated. To assess the extent of variation across PCTs, the coefficient of variation (standard deviation divided by mean) was calculated [11]. Stepwise linear regression was used to assess the relative contribution of potential explanatory factors (social deprivation at PCT level, smoking prevalence at PCT level, proportion of people from Asian ethnic groups, proportion of people from black ethnic groups and amputation rate in adults without diabetes) to the variation in total and major amputation rates in adults with diabetes.

\section{Results}

In the 3 year period between 1 April 2007 and 31 March 2010 there were 34,109 lower limb amputations (major and minor operations combined) in adults in England. Of these $16,693(48.9 \%)$ were in people in whom diabetes had been coded as a co-morbidity — giving an incidence rate in diabetes of 2.51 per 1,000 person-years compared with 0.11 per 1,000 person-years in people without diabetes (relative risk of 23.3). There were 10,216 minor amputations and 6,477 major amputations in people with diabetes, equating to 1.57 and 0.99 per 1,000 person-years, respectively. A smaller proportion of amputations in people with diabetes were above the ankle compared with people without diabetes $(38.8 \%$ vs $47.9 \%$, $\chi^{2}=563.14, p<0.0005$ ).

There was an eightfold variation across PCTs in England in the incidence of total (major and minor combined) 
amputation rates in people with diabetes (range 0.64-5.16 per 1,000 person-years; $\mathrm{CV}=0.328$ ). The range of variation in total amputation rates in people without diabetes was similar (0.03-0.24 per 1,000 person-years; $\mathrm{CV}=0.365)$. The variation between PCTs in the incidence of major amputations in diabetes was tenfold $(0.22-2.20$ per 1,000 person-years; $\mathrm{CV}=0.365$; see Figs 1,2 ), and was similar to that for minor amputations $(0.30-3.25$ per 1,000 person-years; $\mathrm{CV}=0.369)$. There was greater variation in the incidence of major amputation in people without diabetes $(0.01-0.16$ per 1,000 person-years; $C V=0.405$, see Fig. 3 ). The incidence of minor and major amputations in people with diabetes were positively correlated ( $r=0.537, p<0.0005$, see Fig. 4$)$ and a similar correlation was found in people without diabetes $(r=0.611, p<0.0005$; see Fig. 5). At the PCT level the ratio of major to minor amputations ranged from 0.19 to 2.17 for people with diabetes and from 0.30 to 2.50 for those without diabetes ( $\mathrm{CV}=0.377$ and 0.387 , respectively). Furthermore, amputation rates in adults with diabetes were correlated with amputation rates in adults without diabetes $(r=$ $0.433, p<0.0005$ for all amputations, see Fig. 6 , and $r=0.528$, $p<0.0005$ for major amputations, see Fig. 7). No univariate association was found between the incidence of total amputations in people with diabetes and PCT-level data on either average social deprivation $(r=-0.138, p=$ $0.092)$ or smoking prevalence $(r=0.137, p=0.096)$. The incidence of amputations in people with and without diabetes was negatively correlated with the proportion of the population from Asian ethnic groups and black ethnic groups (see Table 1).

Regression analysis showed that among adults with diabetes the only factors found to be independently associated with variation in amputation rates were the amputation rate in adults without diabetes and the proportion of the population from Asian ethnic groups (total amputations, $F=22.632$, adjusted $r^{2}=0.226, p<0.0005$; major amputations, $F=34.692$, adjusted $r^{2}=0.313, p<0.0005$ ).

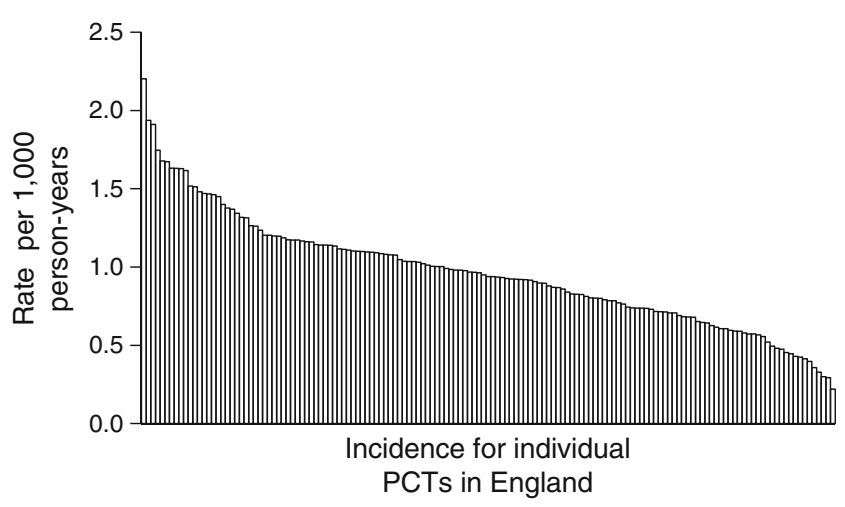

Fig. 1 Variation in the incidence of major amputation in adults with diabetes between PCTs in England 2007-2010, expressed per $1 \times 10^{3}$ population with diabetes

\section{Discussion}

Principal findings These data confirm that diabetes continues to confer a very high relative risk of amputation. They also reveal that in both people with diabetes and without diabetes there is a tenfold variation between PCTs in the incidence of major amputation and an eightfold variation in the incidence of all amputations (major and minor combined). Furthermore, positive correlations between the incidence of minor and major amputations were observed in people both with diabetes and without diabetes. Finally, the data also provide evidence that health economies with a high incidence of amputations in people with diabetes also tended to have a high incidence of amputations in people without diabetes. Variation in the incidence of amputations in people without diabetes explained $18.8 \%$ of the variation in incidence of total amputations and $27.9 \%$ of the variation in major amputations in people with diabetes. No correlation was observed at the PCT level between the incidence of amputation in diabetes and either tobacco use or social deprivation. There was a negative correlation between the incidences of both total and major amputation and estimates of the PCT prevalence of both black and Asian racial groups, and this remained significant for Asians after regression analysis. The potential contribution of race reinforces earlier data suggesting that the incidence of amputation is lower in both Asian [12, 13] and black [14] minorities in UK.

Documentation of wide geographical variation in amputation incidence within a single nationalised healthcare system is of great potential importance. Access to care should not differ and so variation might point to key differences in the organisation or delivery of care.

Strengths and weaknesses of the study Data relating to the incidence of amputation in diabetes have hitherto been difficult to obtain because of the absence of reliable information on the prevalence of diabetes in the community. Since 2004, however, there have been financial incentives within the QOF contract for general practices in England to document the prevalence of diabetes and this has resulted in better information on disease prevalence at the community level. And although there have also been historical concerns about considerable under-ascertainment of diabetes in the hospital discharge coding which forms the basis of HES, the enhanced national treatment tariffs for diabetes-related amputations introduced in the UK over the past decade mean that there is now a financial driver for hospitals to ensure that when diabetes is present it is coded as a comorbidity. It follows that the reliability with which the diagnosis of diabetes can be linked with major and minor amputations in HES and the precision with which the diagnosis of diabetes is known in the English population is increasing but is still likely to be imperfect. 
Fig. 2 Regional variation in England in the incidence of major amputation in diabetes, expressed per $1 \times 10^{3}$ population with diabetes. Contains Ordnance Survey data (C) Crown copyright and database right 2011. Image reproduced with kind permission from Yorkshire and Humber Public Health Observatory
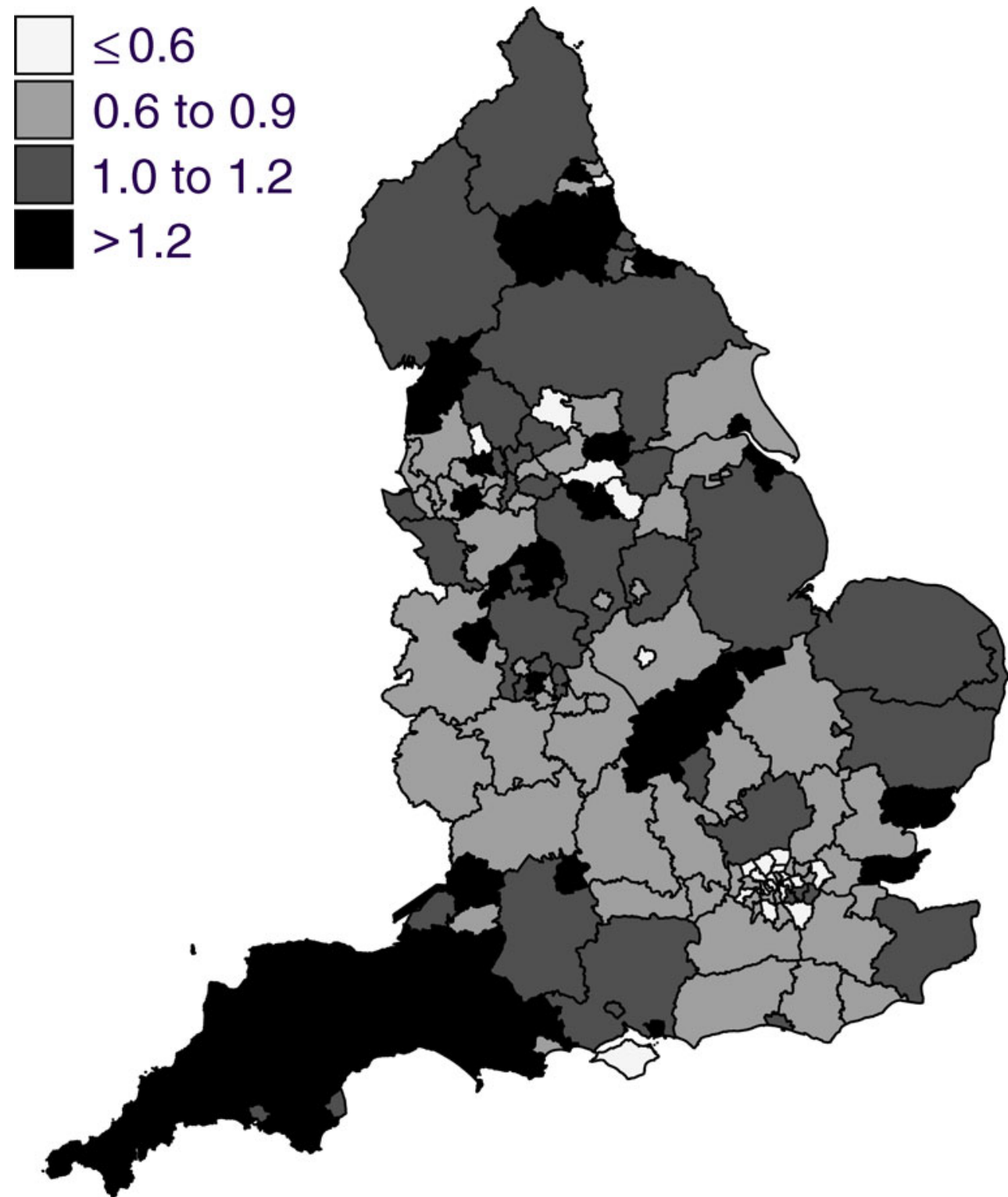

Confidence in these data is supported by their similarity to those obtained by the National Diabetes Audit (NDA) in England and Wales [15], which uses different data sources and does not rely on hospital coding of diabetes. Thus, the rate of total (major and minor combined) amputation in the present study was 2.57 per 1,000 people with diabetes, and this compares with a figure of 2.30 obtained by the NDA. Minor discrepancies could be attributable to differences in the numerators used in the two methods. The present study gathered information on all hospital admissions in which an amputation was undertaken and this will inevitably lead to double counting when the same person has two or more admissions in which one or more amputations were performed. In contrast, the NDA data-which relies on the identification of people with diabetes from general practice records - counts as the numerator only those who have had any (one or more) amputation in the preceding year.
A decision was made to include all amputations (including those precipitated by trauma and malignancy) in this studypartly because the incidence of amputations undertaken for these reasons are proportionately low. Moreover, it could be argued that an amputation undertaken following trauma could have been made necessary because of the co-existence of peripheral arterial disease with or without diabetes. It was for these reasons that traumatic amputations were included in the national study recently completed in France [16].

Strengths and weaknesses in relation to other studies The very large study undertaken by Wrobel and colleagues showed an 8.6-fold variation in the incidence of major amputation in the USA [5]. It might be argued that in the USA social deprivation and variation in access to primary medical care could contribute to such variation but that should not apply in a country such as England in which 


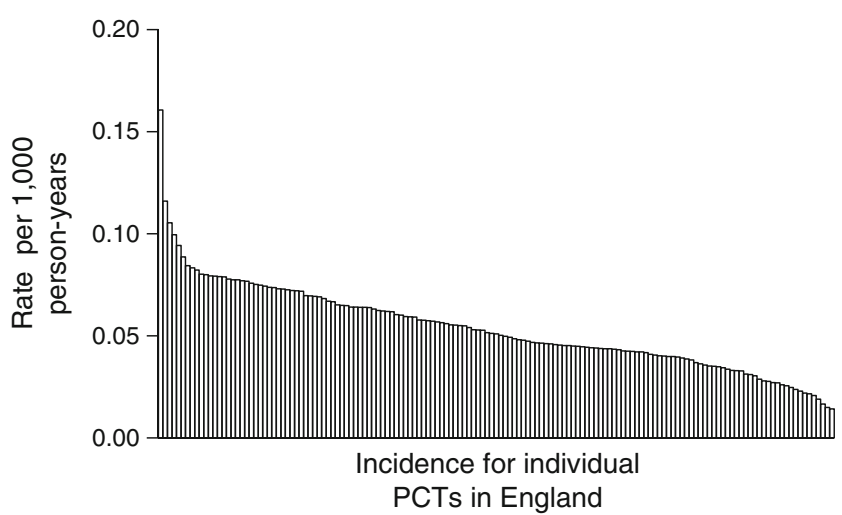

Fig. 3 Variation in the incidence of major amputation in people without diabetes between PCTs in England 2007-2010, expressed per $1 \times 10^{3}$ total population

most of the population is treated by a nationalised health service. And indeed social deprivation at PCT level was not an explanatory variable in our data.

Wrobel and colleagues [5] found no correlation between the incidence of major amputation in their US diabetes and non-diabetes populations, whereas these English data suggest that a positive association exists between diabetes-related and non-diabetes-related amputations. This difference might indicate that in England there may be healthcare organisational variations shared across health economies that have a similar influence on diabetes-related and non-diabetes-related amputations, but this does not apply to a similar extent in the USA.

In the USA it has been argued that the optimal use of minor amputation will lead to prevention of major amputation so that an inverse relationship should be observed between the two types of operation (the 'Hi-Lo' ratio) [17]. There is no evidence that such a relationship exists in England: the correlation between minor and major amputation is positive in both those with and without diabetes: PCTs with a high incidence of minor amputations tend to have a high incidence of major amputations.

One other study has explored variation in the incidence of amputation between different centres in England. Chaturvedi

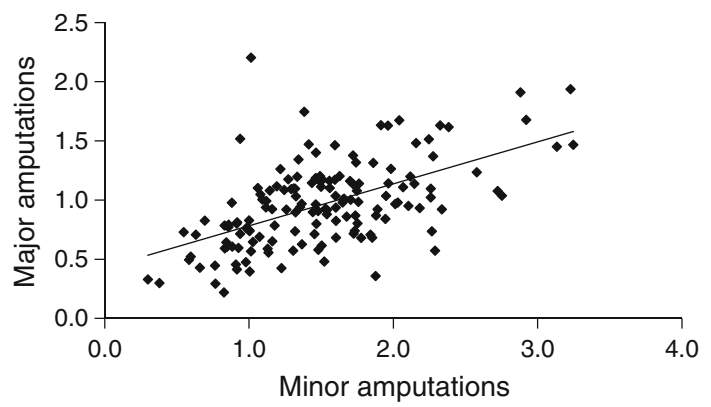

Fig. 4 Correlation between the incidences of major and minor amputations in people with diabetes between PCTs in England 2007-2010. $R=0.537, p=0.0005$

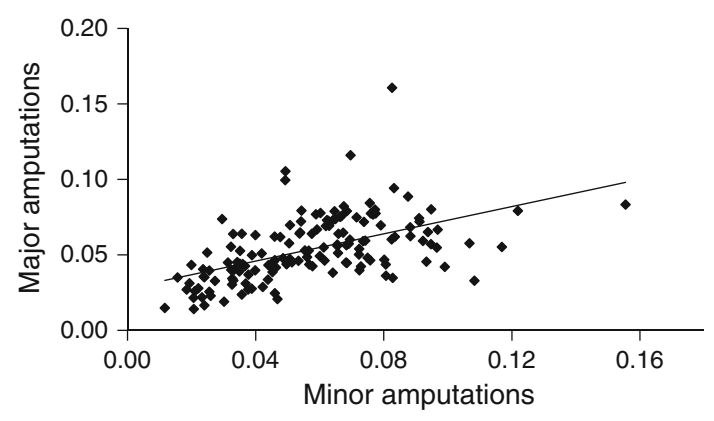

Fig. 5 Correlation between the incidences of major and minor amputations in people without diabetes between PCTs in England 2007-2010. $R=0.611, p=0.0005$

and colleagues included data from four different communities in the Midlands and North of England in the Global Lower Extremity Amputation Study [6], which was a multinational study in the 1990s of the incidence of amputations (major plus minor operations combined also with unoperated gangrene) undertaken. This study reported fourfold variation in the incidence of total diabetes-related amputation (plus gangrene) between the four English centres. A subsequent investigation involving three of these centres concluded that one factor contributing to the observed variation may have been the professional beliefs of the treating clinicians [18].

Implications of the results of the present study Care must be exercised when drawing inferences from observations based on the incidence of amputation, and automatically using them as a marker of the quality of managing established foot disease [19]. In practice, a high incidence of limb loss may variously reflect the extent of the national disease burden, social and religious attitudes leading to delayed presentation, and the availability of good medical care. It must also be remembered that an amputation is a treatment, and treatments are selected on the basis of individual need. If it is judged that a chronic wound on the foot will never heal, then early amputation of the lower leg is often the best option. On the other hand, it may be decided that the overall frailty of the patient, or their co-morbidity or lack of mobility

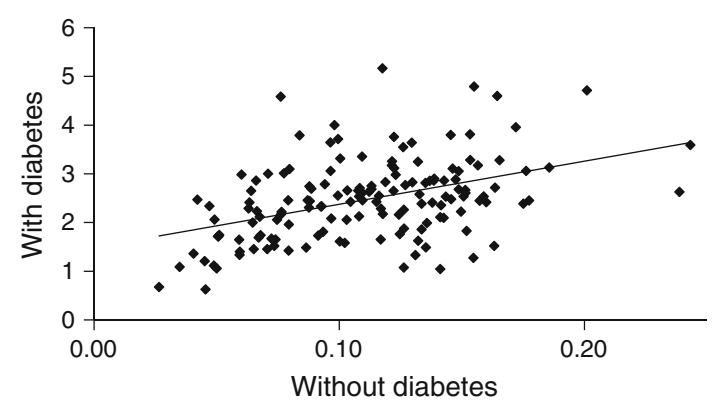

Fig. 6 Correlation between the incidences of total (major and minor combined) amputations in people with and without diabetes between PCTs in England 2007-2010. $R=0.433, p=0.0005$ 


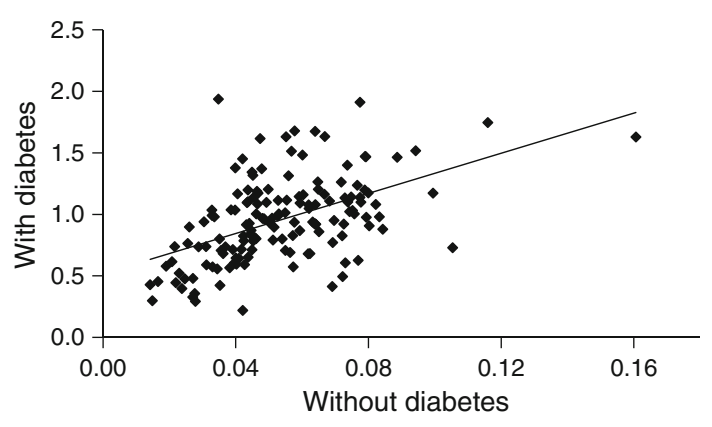

Fig. 7 Correlation between the incidences of major amputations in people with and without diabetes between PCTs in England 2007-2010. $R=0.528, p=0.0005$

is such that amputation is not in their best interests. It is for reasons such as these that a high incidence of amputation does not necessarily reflect adversely on the quality of expert services.

It may also be argued that in people with diabetes the incidence of amputation is more likely to reflect aspects of glucose and vascular-risk-factor control in the years following (or, in the case of type 2 diabetes, even preceding) diagnosis, as effective management will reduce the incidence of neuropathy and peripheral arterial disease which are the main factors predisposing to ulceration and, in turn, to amputation. The implication of this argument is that the person who presents with a foot ulcer already has disease that is far advanced and that the options available to a specialist team to save the limb are relatively limited. Despite this, there are a number of careful studies that have shown that it is possible to reduce the incidence of amputation in diabetes by implementing changes to the structure of diabetes foot ulcer care. In England, in particular, two centres have reduced the incidence of major amputation by a factor of four to five times by creating an expert multidisciplinary service where none previously existed [20,21]. Results such as these suggest

Table 1 Correlation between incidence of amputations and proportion of population from South Asian and black ethnic groups at the PCT level

\begin{tabular}{|c|c|c|c|c|}
\hline \multirow[t]{3}{*}{ Population } & \multicolumn{4}{|c|}{$\begin{array}{l}\text { Proportion of the population from ethnic } \\
\text { groups }\end{array}$} \\
\hline & \multicolumn{2}{|l|}{ Asian } & \multicolumn{2}{|l|}{ Black } \\
\hline & $r$ & $p$ value & $r$ & $p$ value \\
\hline \multicolumn{5}{|l|}{ People with diabetes } \\
\hline Total amputations & -0.423 & $<0.005$ & -0.351 & $<0.005$ \\
\hline Major amputations & -0.429 & $<0.005$ & -0.469 & $<0.005$ \\
\hline \multicolumn{5}{|c|}{ People without diabetes } \\
\hline Total amputations & -0.617 & $<0.005$ & -0.741 & $<0.005$ \\
\hline Major amputations & -0.480 & $<0.005$ & 0.640 & $<0.005$ \\
\hline
\end{tabular}

strongly that even in the presence of advanced lower limb disease the incidence of amputation can be reduced, and reduced quite considerably if the baseline incidence is higher than expected, by changing working practice. It follows that the wide variation in incidence reported here may, to some extent, have its origins in lack of consistency in the delivery of care; if so, this may explain also the variation known to persist between many countries worldwide [6, 18, 22]. If the observations made in the earlier study by Connelly and colleagues [18] also apply, then there may be differences in professional opinion and belief that contribute to higher and lower incidence of amputation and perhaps it is these differences that are minimised by multidisciplinary team working.

If the observed variation between PCTs in the incidence of amputation does reflect differences in the local structures of care including the varying involvement and/or organisation of an expert multidisciplinary team in the management of limb-threatening disease, this has major implications for planning foot care services for people with or without diabetes, both in England and in other countries. Every person with lower limb disease should have access to the same clinical expertise, and inclusion of all the necessary skills inevitably means multidisciplinary team work. For people with diabetes the need for multidisciplinary teams to be established throughout the country has been emphasised in the UK via the Putting Feet First report [23] and the updated version of the National Minimum Skills Framework [24] recently published by Diabetes UK and NHS Diabetes, as well as the 2011 guidelines on the management of established foot disease in hospitals published by the National Institute for Health and Clinical Excellence [25]. This study supports the hope that universal implementation of such structures will lead to a reduction in the current substantial level of geographical variation in the incidence of amputation, with a resultant reduction in the incidence overall.

If aspects of professional belief and individual practice contribute to the variations observed in the present study, it might explain the associations observed between the incidences of major and minor amputations and between people with and without diabetes. Alternatively these associations might be explained by shared factors related to the organisation of care in different PCTs. The existence of these associations implies that these findings should trigger a search for structural and organisational factors critical to the outcome of severe foot disease, with special relevance to diabetes.

Further research Attempts should be made to determine how the structure and organisation of care varies and whether previously identified markers of care quality (such as multidisciplinary team working and speed of referral to specialist services) correlate with the incidence of amputation. Studies also need to be undertaken in which the incidence of 
amputation is not used as a marker of disease management in isolation, but combined with measures of well-being and social function.

Acknowledgements The authors would like to thank S. Jones, Diabetes Health Intelligence, Yorkshire and Humber Public Health Observatory, for her work on extracting and collating the HES data. We also thank G. Rayman for helpful discussion.

Duality of interest The authors declare that there is no duality of interest associated with this manuscript

Contribution statement NH, RJY and WJJ contributed to the study design and all three were closely involved in the production of the manuscript and agreed its final form. NH undertook all the analyses.

\section{References}

1. Boulton AJ, Vileikyte L, Ragnarsson-Tennvall G, Apelqvist A (2005) The global burden of diabetic foot disease. Lancet 366:1719-1724

2. Ali SM, Fareed A, Humail SM et al (2008) The personal cost of diabetic foot disease in the developing world - a study from Pakistan. Diabet Med 25:1231-1233

3. Margolis DJ, Allen-Taylor L, Hoffstad O, Berlin JA (2002) Diabetic neuropathic foot ulcers: the association of wound size, wound duration, and wound grade on healing. Diabetes Care 25:1835-1839

4. Ince P, Kendrick D, Game F, Jeffcoate W (2007) The association between baseline characteristics and the outcome of foot lesions in a UK population with diabetes. Diabet Med 24:977-981

5. Wrobel JS, Mayfield JA, Reiber GE (2001) Geographic variation of lower-extremity major amputation in individuals with and without diabetes in the Medicare population. Diabetes Care 24:860-864

6. Chaturvedi N, Stevens LK, Fuller JH, Lee ET, Lu M (2001) Risk factors, ethnic differences and mortality associated with lowerextremity gangrene and amputation in diabetes. Diabetologia 44 (Suppl 2):S65-S71

7. NHS Right Care [2010]. The NHS atlas of variation in healthcare: reducing unwarranted variation to increase value and improve quality. Available from www.rightcare.nhs.uk/atlas/, accessed 5 September 2011

8. The Information Centre for Health and Social Care (2008) Model based estimates of healthy lifestyle behaviours at PCO level 20032005. Available from www.ic.nhs.uk/statistics-and-data-collections/ population-and-geography/neighbourhood-statistics/neighbourhoodstatistics:-model-based-estimates-of-healthy-lifestyle-behaviours-atpco-level-2003-05, accessed 5 September 2011

9. Noble M, McLennan D, Wilkinson K, Whitworth A, Barnes H, Dibben C (2008) The English indices of deprivation 2007.
Available from www.communities.gov.uk/publications/communities/ indiciesdeprivation07, accessed 5 September 2011

10. Population estimates by ethnic group (2011) Available from www. ons.gov.uk/ons/publications/re-reference-tables.html?edition=tcm \%3A77-50032, accessed 21 November 2011

11. Appleby J, Raleigh V, Frosini F, Bevan G, Gao H, Lyscom T (2011) Variations in health care: the good, the bad and the inexplicable. The Kings Fund, London

12. Chaturvedi N, Abbott CA, Whalley P, Leggetter SY, Boulton AJ (2002) Risk of diabetes-related amputation in South Asians vs. Europeans in the UK. Diabet Med 2002:99-104

13. Abbott CA, Chaturvedi N, Mailk RA et al (2010) Explanations for the lower rates of diabetic neuropathy in Indian Asians versus Europeans. Diabetes Care 33:1325-1330

14. Leggetter S, Chaturvedi N, Fuller JH, Edmonds ME (2002) Ethnicity and risk of diabetes-related lower extremity amputation: a population-based, case-control study of African Caribbeans and Europeans in the United Kingdom. Arch Intern Med 162:73-78

15. National diabetes audit (2010) Available from www.ic.nhs.uk/ webfiles/Services/NCASP/audits\%20and\%20reports/7121 National $\% 20$ Diabetes\%20Audit_final.pdf, accessed 28 August 2011

16. Fosse $\mathrm{S}$, Hartemann-Heurtier A, Jacqeminet $\mathrm{S}$, van $\mathrm{Ha} \mathrm{G}$, Grimaldi A, Fagot-Campagna A (2009) Incidence and characteristics of lower limb amputations in people with diabetes. Diabet Med 26:391-396

17. Wrobel JS, Robbins J, Armstrong DG (2006) The high-low amputation ratio: a deeper insight into diabetic foot care? J Foot Ankl Surg 45:375-379

18. Connelly J, Airey M, Chell S (2001) Variation in clinical decision making is a partial explanation for geographical variation in lower extremity amputation rates. Br J Surg 88:529-535

19. Jeffcoate WJ, van Houtum WH (2004) Amputation as a marker of the quality of foot care in diabetes. Diabetologia 47:2051-2058

20. Krishnan S, Nash F, Baker N, Fowler D, Rayman G (2008) Reduction in diabetic amputations over 11 years in a defined U.K. population: benefits of multidisciplinary team work and continuous prospective audit. Diabetes Care 31:99-101

21. Canavan RJ, Unwin NC, Kelly WF, Connolly VM (2008) Diabetes- and nondiabetes-related lower extremity amputation incidence before and after the introduction of better organized diabetes foot care: continuous longitudinal monitoring using a standard method. Diabetes Care 31:459-463

22. Moxey PW, Gogalniceanu P, Hinchliffe RJ et al (2011) Lower extremity amputations - a review of global variability in incidence. Diabet Med 28:1144-1153

23. Putting feet first: commissioning specialist services for the management and prevention of diabetic foot disease in hospitals (2011) Available from www.diabetes.org.uk/Documents/Reports/ Putting_Feet_First_010709.pdf, accessed 28 August 2011

24. Putting feet first: national minimum skills framework (2011) Available from www.diabetes.org.uk/Documents/Professionals/ Education\%20and\%20skills/NMSF_16Feb2011.pdf, accessed 28 August 2011

25. Diabetic foot problems - inpatient management (2011) Available from www.nice.org.uk/CG119, accessed 28 August 2011 